\title{
Acute effects of electromagnetic stimulation of the brain on cortical activity, cortical blood flow, blood pressure and heart rate in the cat: an evaluation of safety
}

\author{
J A Eyre, P A Flecknell, B R Kenyon, T H H G Knh, S Miller
}

\begin{abstract}
The influence of repeated high intensity electromagnetic stimulation of the brain on cortical activity, cortical blood flow, blood pressure and heart rate has been investigated in the cat, to evaluate the safety of the method. The observations have been made in preparations under propofol anaesthesia before, during and after periods of anoxia. Electromagnetic stimulation of the brain evoked activity in descending motor pathways and was recorded by activity in the median nerve and by muscle twitches. Following repeated series of high intensity stimulation there were no systematic changes in somatosensory evoked potentials or background EEG, nor were there signs of epileptogenic activity during electromagnetic stimulation, before, during or after periods of anoxia. No systematic changes in cortical blood flow, blood pressure or heart rate were observed during electromagnetic stimulation, before or after periods of anoxia. In conclusion, no acute adverse consequences following electromagnetic stimulation in the normal and anoxic cat brain were demonstrated.
\end{abstract}

Electromagnetic stimulation of the human motor cortex to evoke motor action potentials in skeletal muscle has developed into a widely used non-invasive technique for evaluating the function of descending motor pathways to the spinal cord and brain stem. ${ }^{1}$ Normal subjects and subjects suffering from selected neurological conditions, ranging in age from preterm babies to elderly adults, have received repeated electromagnetic stimulation of the brain with magnetic fields lasting $<300 \mu$ s and with a flux of up to 2 Tesla. ${ }^{2-11}$ In studies so far reported neither acute nor longer term harmful side effects have been observed in any of the subjects. It may be argued that the criteria for harmful side effects are relatively crude (for example, fits, headache, gross cognitive loss, exacerbation of existing neurological signs) and that more subtle changes of neuronal function or neurovascular control might result from electromagnetic stimulation of the brain.

The aim of this study was therefore to determine in an animal model if electromag- netic stimulation of the brain might evoke transitory or longer lasting changes of blood flow and neuronal activity locally within the cerebral cortex. Since motor action potentials following brain stimulation have been studied in subjects suffering from neurological conditions involving cerebral ischaemia or anoxia, ${ }^{911}$ it also seemed important to investigate whether the presence of cerebral anoxia in any way changed or compounded acute responses to electromagnetic stimulation. Finally, heart rate and blood pressure were recorded as indices of general homeostatic responses and as reference points for interpreting changes in cortical blood flow. The results have been reported briefly. ${ }^{12}$

\section{Methods}

The experiments were performed on five cats weighing between $3 \cdot 1$ and $4.3 \mathrm{~kg}$. Surgical anaesthesia was induced with propofol (Diprivan, ICI) given intravenously $7 \cdot 5$ $\mathrm{mg} \cdot \mathrm{kg}^{-1} \cdot \mathrm{hr}^{-1}$. and maintained with a continuous intravenous infusion of propofol 15-30 $\mathrm{mg} \cdot \mathrm{kg}^{-1} \cdot \mathrm{hr}^{-1}$. After completion of the surgical procedures neuromuscular block was obtained with Pancuronium (Pavulon, OrganonTetrika) $0.06 \mathrm{mg} \cdot \mathrm{kg}^{-1}$. The infusion of propofol was continued throughout the period of neuromuscular block and the level of anaesthesia monitored by heart rate and blood pressure.

A diagram of the stimulating and recording arrangements is shown in fig 1A. The trachea was intubated and intermittent positive pressure ventilation established with nitrous oxide and oxygen, with a $\mathrm{FiO}_{2}$ of $40 \%$. A $1.2 \mathrm{~mm}$ external diameter Portex cannula was inserted into the left femoral artery and advanced into the aorta for measuring arterial blood pressure. The electrocardiogram (ECG) was recorded from surface electrodes applied proximally to the left and right forelimbs and to the right hindlimb. In the right forelimb the ulnar and median nerves were exposed 2-4 $\mathrm{cm}$ below the elbow and their cut central ends drawn into tunnel electrodes. ${ }^{13} \mathrm{~A}$ craniotomy was made to expose the left sensorimotor cortex. The electroencephalogram (EEG) was recorded from the somatosensory area for the forelimb ${ }^{14}$ using a $\mathrm{Ag} / \mathrm{AgCl}$ ball electrode $(0 \cdot 5$ $\mathrm{mm}$ diameter) which was placed in contact with the surface of the brain and suspended on a flexible coil of silver wire. Two $\mathrm{Ag} / \mathrm{AgCl}$ 
Figure 1 Experimental method. A, stimulating and recording

arrangements. $B$.

Magnetic field intensity determined with a $10 \mathrm{~mm}$ diameter, 2 turn, circular search coil at varying distances from centre of stimulating coil: starred symbols, at distances along axis at right angles to plane of coil; squares, along central plane of coil. triangles, along plane of coil $2 \mathrm{~cm}$ below level of central plane.
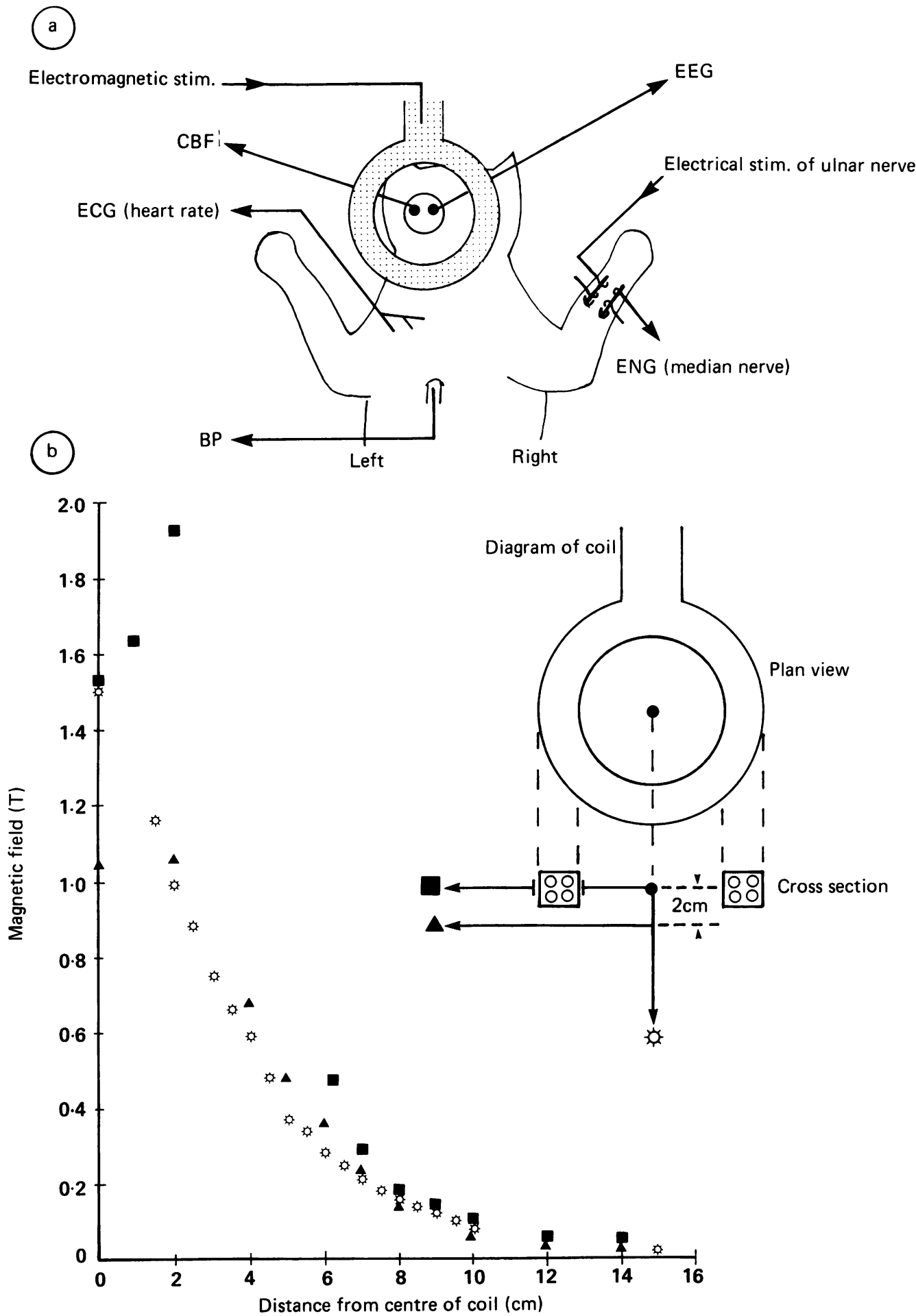

wires were inserted into the neck muscles to act, respectively, as an indifferent recording electrode and an earth electrode. A fibre-optic probe was placed on the corresponding motor area of the forelimb for the continuous measurement of cortical blood flow (CBF) by laser Doppler spectroscopy. ${ }^{15}$ This method provides a particularly sensitive measure of cortical blood flow as it records flow in capillaries. Electromagnetic stimulation of the brain was delivered by the apparatus described by Byrne et al. ${ }^{16}$ The coil was circular, mean diameter $9 \mathrm{~cm}$, and contained 9 turns of wire wound in a helix. Pulsed magnetic fields of up to 1.9 Tesla with a rise time of $100 \mu \mathrm{s}$ and a total duration of $300 \mu \mathrm{s}$ were used. Measurements at the bench (using a 2 turn, circular search coil, $10 \mathrm{~mm}$ diameter) showed the magnetic field at the surface of the brain (that is, $2 \mathrm{~cm}$ below the level of the central plane) would be maximal along the axis through the centre of the coil perpendicular to the winding (fig 1B). The stimulating coil was therefore placed tangential to the vault of the skull with the geometric centre (axis) overlying the cruciate sulcus.

Using the method by Barker et al $^{17}$ the current density in the scalp is calculated as 
$20.1 \mathrm{~mA} . \mathrm{cm}^{-2}$ per phase (ph) and the charge density as $2 \cdot 3 \mu \mathrm{C} . \mathrm{cm}^{-2}$.ph. For the surface of the brain, taken at $2 \mathrm{~cm}$ below the coil (see fig 1), the magnetic field is $1 \cdot 2$ Tesla (starred symbol); the current density is $4 \cdot 2$ $\mathrm{mA} . \mathrm{cm}^{-2} \cdot \mathrm{ph}$ and the charge density 0.43 $\mu$ C.cm ${ }^{-2}$.ph.

Signals recorded from the median nerve were amplified by $80 \mathrm{~dB}$ and filtered with a $-3 \mathrm{~dB}$ bandpass of $100 \mathrm{~Hz}$ to $10 \mathrm{kHz}$. The EEG was amplified by $80 \mathrm{~dB}$, filtered with -3 $\mathrm{dB}$ points of 1 and $100 \mathrm{~Hz}$ and recorded continuously throughout the experiment. Sensory evoked potentials (SEP) were recorded following electrical stimulation of the ulnar nerve with pulses of $0.1 \mathrm{~ms}$ at approximately twice the threshold for evoking a short latency (spinal) reflex response in the median nerve. The inputs of the amplifier circuits recording the EEG and ENG were switched and disconnected from the preparation for between 1-3 ms following each magnetic stimulus to minimise induced electrical artefact. $^{18}$

In each experiment baseline recordings were made of EEG, CBF, blood pressure, ECG and instantaneous heart rate. Averages of 20 SEPs were computed with a sampling duration of $40 \mathrm{~ms}$ and at a stimulus rate of 1 per 5s. Electromagnetic stimuli at maximum intensity (see above) were then applied every $5 \mathrm{~s}$ in runs of 10 to 50 giving totals of 120 to 500 stimuli. The runs were separated by intervals of one to 10 minutes to allow the coil to cool. ENG, EEG, CBF, blood pressure and heart rate were recorded continuously. SEPs were evoked in an interleaved manner $2.5 \mathrm{~s}$ after each brain stimulus. To explore the effect of cerebral anoxia, the preparation was then ventilated for five minutes with $100 \%$ $\mathrm{NO}_{2}$ and thereafter returned to $30-50 \%$ oxygen. When the preparation appeared stable as judged by blood pressure and heart rate, further runs of interleaved electromagnetic brain stimulation and electrical ulnar nerve stimulation were applied. Measurements were continued for up to 75 minutes after the first period of anoxia.

The data were recorded on to paper for immediate scrutiny and on to magnetic tape for off-line analysis.

\section{Results}

Neural responses evoked by stimulation

In all five experiments electromagnetic stimulation of the brain evoked activity in the right median nerve with a latency of $12-15 \mathrm{~ms}$ and a variable duration at times exceeding $30 \mathrm{~ms}$ (fig 2C). The latency of the responses evoked in the median nerve did not change systematically following prolonged series of maximum intensity electromagnetic stimulation. In one experiment electromagnetic stimulation was applied before the onset of neuromuscular blockade and twitches were observed in the right forelimb muscles.

Evoked responses were recorded from the cerebral cortex with the electrode placed over the forelimb zone of the right somatosensory area (fig 2A and B). The earliest response occurred at $10 \mathrm{~ms}$ (arrow in fig 2B) and continued with variable longer latency responses up to $250 \mathrm{~ms}$ (fig $2 \mathrm{~A}$ ).

In all experiments electrical stimulation of the ulnar nerve evoked activity in the somatosensory area of the cerebral cortex with a latency of $7.5 \mathrm{~ms}$ (fig $2 \mathrm{E}$ ), followed by variable longer latency responses up to about $250 \mathrm{~ms}$ (fig 2D). Responses were also observed in the median nerve at a latency of $7.5 \mathrm{~ms}$, consistent with that of a spinal reflex (fig $2 F$ ).

\section{EEG before and after electromagnetic stimulation} Figure 3 shows representative sections of EEG recorded in one experiment, A before, and B and $C$ during, electromagnetic stimulation. Averages of 20 SEPs computed before, and in an interleaved sequence during, electromagnetic stimulation are shown inserted above each of the EEG traces. No systematic changes in background EEG activity nor in the SEP recordings were observed following repeated maximum intensity electromagnetic stimulation before anoxia. In figs $3 D, E$ and $F$ comparable EEG and SEP recordings obtained after a five minute period of anoxia are shown. After the period of anoxia the background EEG became of lower voltage and the amplitudes of the SEPs and the cortical responses to electromagnetic stimulation were smaller (compare figs $3 \mathrm{~B}$ and $3 \mathrm{D}$ ). There were no systematic changes in background EEG during electromagnetic stimulation after anoxia; the amplitude of the SEP increased with time during electromagnetic stimulation until the amplitude returned to pre-anoxic levels. There was no evidence in the EEG of epileptogenic activity. Similar results were obtained in all experiments.

Influence of electromagnetic stimulation on blood pressure, cortical blood flow, ECG, and heart rate before and after periods of anoxia

The traces in fig 4 illustrate recordings of blood



Figure 2 Responses to electromagnetic stimulation of the brain and to electrical stimulation of the right ulnar nerve. Responses, evoked by electromagnetic stimulation, in the left sensorimotor cortex $(A, B$. Note different time scales.) and in the right median nerve (C). Responses, evoked by electrical stimulation (single shock) of the right ulnar nerve, in the left sensorimotor cortex $(D, E$. Note different time scales) and in the right median nerve $(F)$. 
Figure 3 EEG recordings from sensorimotor cortex, with insets of SEPs averaged from 20 consecutive responses to ulnar nerve stimulation. $A$, sample of control EEG before electromagnetic stimulation and ulnar nerve stimulation. The inset SEP average was made with ulnar nerve stimulation after the control period illustrated in $A . B$ and $C$, samples of $E E G$ obtained at different times during 250 alternate electromagnetic and ulnar nerve stimulations. The numbers in these and subsequent traces indicate the cumulative counts of stimulations. (The ulnar nerve counter was set to 0 after the control SEP in $A$ had been obtained.) $D, E$ and $F$, responses to alternate electromagnetic brain stimulation and electrical stimulation of ulnar nerve following five minutes anoxia.

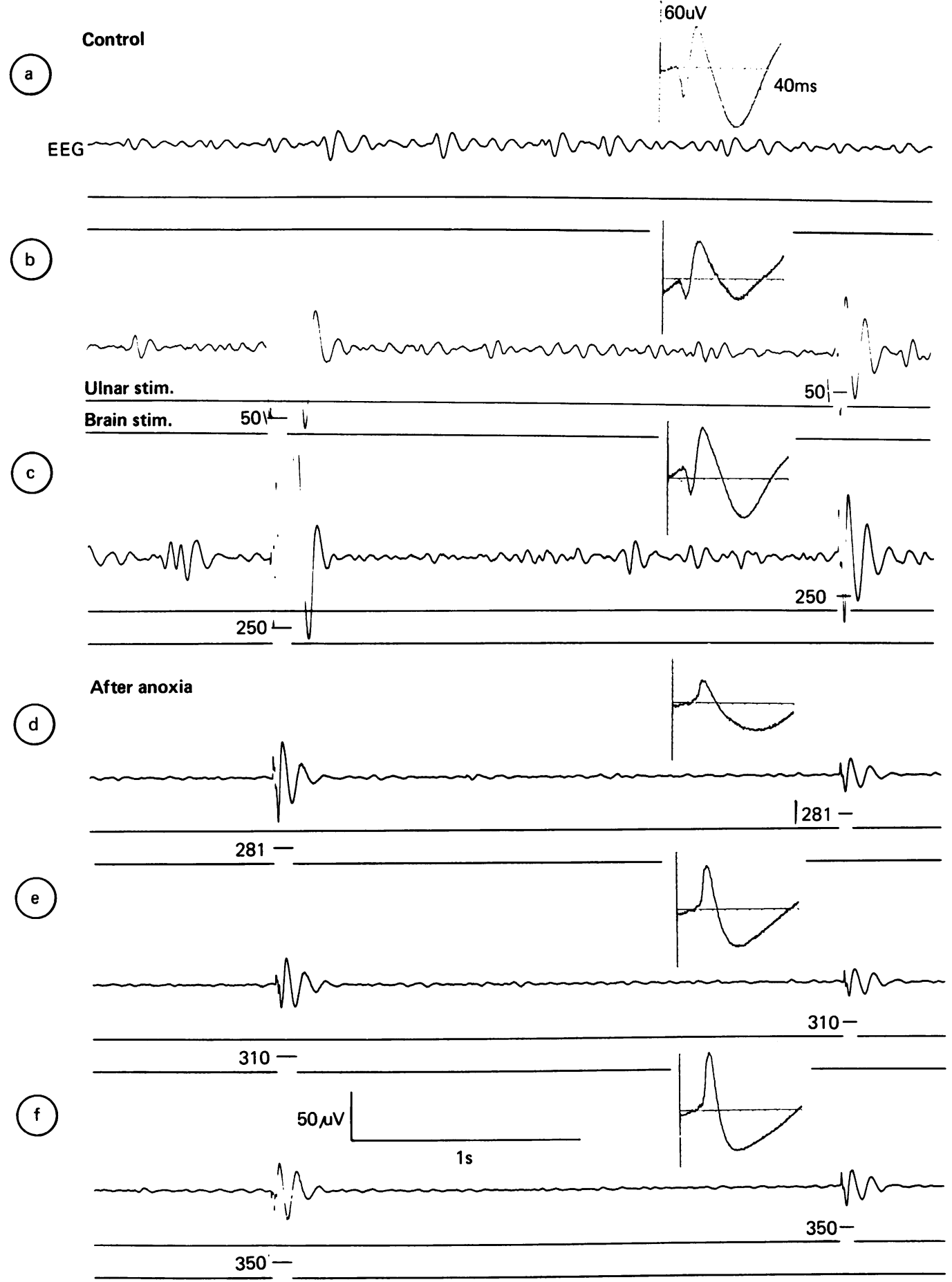

pressure, cortical blood flow, ECG and heart rate, before (A), and after $(B, C, D)$, the onset of maximal electromagnetic stimulation of the cortex. Figures $4 \mathrm{E}, \mathrm{F}$ and $\mathrm{G}$ show comparable recordings of these variables following a five minute period of anoxia. The results are representative of those obtained in all experiments.

The data presented in fig 4 demonstrate that before anoxia no systematic changes in blood pressure occurred following electromagnetic stimulation of the brain. After the period of anoxia the baseline recordings before the onset of electromagnetic stimulation showed that there had been a decrease in blood pressure. Subsequently there was a steady rise in blood pressure to the pre-anoxic level. No acute changes of blood pressure associated with electromagnetic stimulation were observed.

In the $300 \mathrm{~ms}$ following each electromagnetic stimulus changes occur in the output from the laser Doppler flow meter. In fig 5A the trace was obtained from a live brain using the standard experimental recording conditions, with both the laser and its electronic flow meter circuits switched on. The recording in fig 5B was obtained with the laser and its flow meter circuits on, but from the brain 10 minutes after death. When the electromagnetic stimulus is triggered, the current passing through the coil causes the wires forming the conductor to move towards each other. Despite secure fixation of the head of the preparation, the stimulating coil and the fibre optic probe of the laser, it seems 
Figure 4 Influence of electromagnetic brain stimulation on blood pressure, cortical blood flow and instantaneous heart rate. Control period: $A$, before electromagnetic brain stimulation; $B$ and $C$, during electromagnetic stimulation. $D$, control period immediately before anoxia. Following five minutes of anoxia : $E$, before electromagnetic stimulation; $F$ and $G$, during electromagnetic stimulation. Numbers beneath the traces indicate cumulative counts of electromagnetic stimuli.

Figure 5 Cortical blood flow measured under different conditions. $A$, Normal recording with laser circuits switched on and a live preparation. $B$, Laser circuits on but with brain 10 minutes after death. $C$, laser circuits switched off but with live preparation.
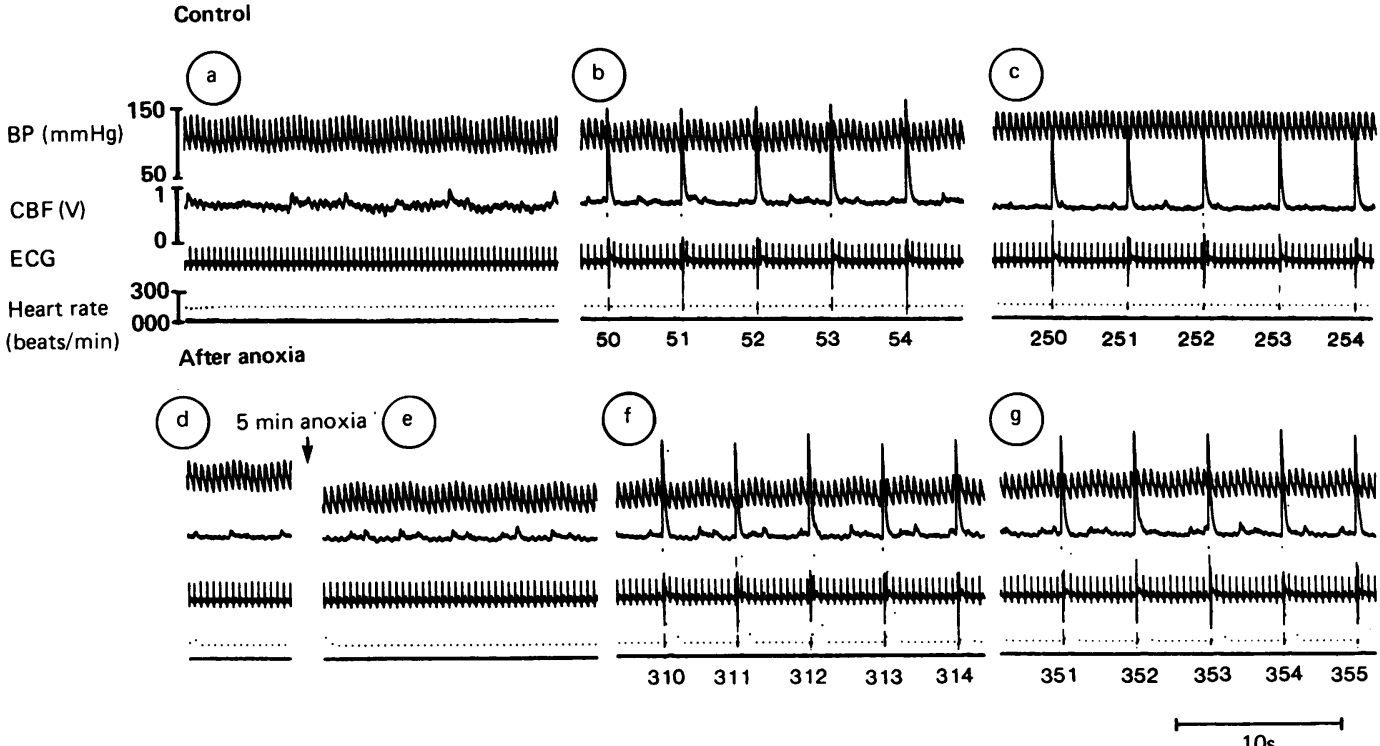

$10 \mathrm{~s}$

likely that mechanical vibration generated by the movements of the conducting wires of the coil were transmitted to the optic fibre probe and represent a component of the transient response shown in fig $5 \mathrm{~A}$. In contrast, in fig $5 \mathrm{C}$ the trace was obtained with the laser switched off, but with the flow meter circuits on and with a live preparation. It is apparent that a further component of the transient change results from currents induced in the flow meter circuits by the pulsed magnetic field.

A change in cortical blood flow during a period of less than $300 \mathrm{~ms}$ following an electromagnetic stimulus could have been masked by the electrical and mechanical artefacts in the flow meter trace, but such a brief variation in cortical blood flow is physiologically improbable. It should be noted that there are no changes in the overall level of cortical blood flow during repeated electromagnetic stimuli, both before and after anoxia (fig 4).

In fig $6 \mathrm{~A}$ transient high frequency breakthrough of the electromagnetic stimulus is evident in the ECG trace. The instantaneous heart rate trace is derived from the ECG and, as a consequence, shows an apparent transient increase. From the ECG trace, however, it can be seen that there is no alteration in the beat to beat $(R-R)$ interval. Detailed analyses of the ECG traces have shown there to be stability of heart rate between electromagnetic stimuli and throughout the period of stimulation (fig 4).

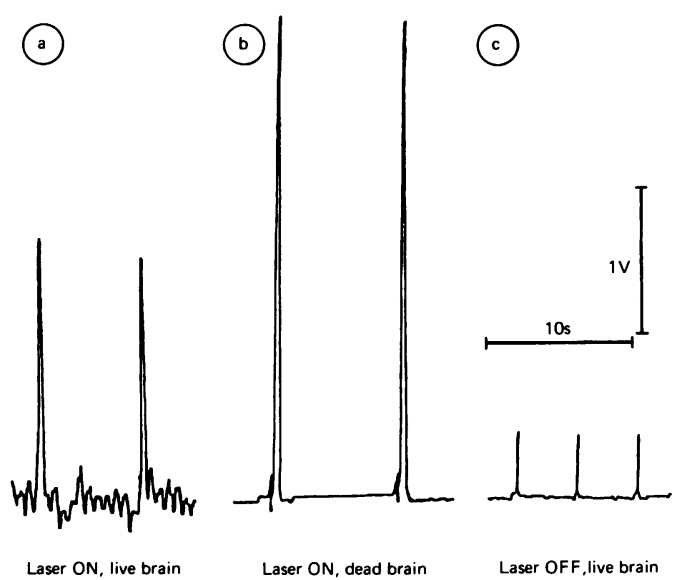

We therefore concluded that there are no systematic changes in heart rate induced by repeated electromagnetic stimulation of the brain.

\section{Electromagnetic stimulation during severe} anoxia

In the traces recorded after a period of anoxia (fig 4D, E and F) the cortical blood flow, despite the initial fall and subsequent rise in blood pressure, remains at the pre-anoxic level. From this it can be concluded that local autoregulation of cortical blood flow was still present. The traces in fig 7 , however, were obtained during severe anoxia which followed two previous five minute periods of anoxia. At this stage autoregulation of cortical blood flow was no longer present and cortical blood flow followed passively the upward and then downward swings of blood pressure. Despite the degree of anoxic damage to the brain and the loss of autoregulation of blood flow, the electromagnetic stimulus did not evoke immediate or longer term changes in cortical blood flow. In the EEG there were no signs of fast wave activity which might signal epileptogenic kindling. As cortical blood flow fell towards zero, the SEPs evoked by stimulation of the ulnar nerve diminished in amplitude and then disappeared. The cortical response to electromagnetic stimulation remained until the end of the recording.

\section{Discussion}

Electromagnetic stimulation of the brain in the cat evoked activity in the cerebral cortex and in descending motor pathways. This is a new observation since the technique has previously only been shown to stimulate the cortex in primates. ${ }^{19} 20$ The onset of the responses in the somato-sensory cortex occurring at $10 \mathrm{~ms}$ would suggest that this cortical area was not being excited directly. It is likely that the responses resulted from activity evoked by projections from other areas of the cerebral cortex excited earlier by the stimulus. Alternatively, the latency of the responses could possibly be associated with looped pathways 


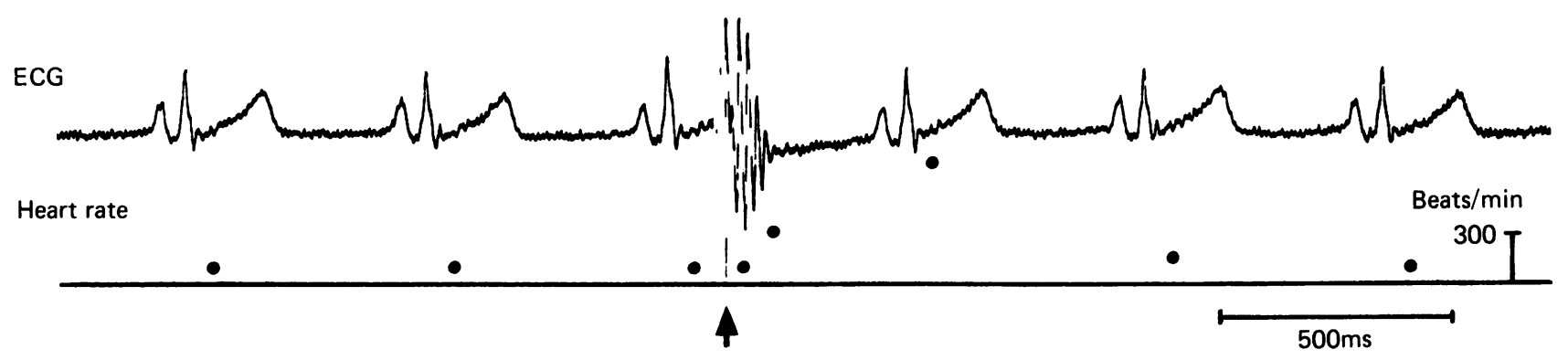

Figure 6 ECG and instantaneous heart rate recordings during electromagnetic stimulation (arrow), demonstrating a false rise in instantaneous heart rate due to break-through in the ECG trace of the electromagnetic stimulus artefact. Note that $R-R$ intervals are regular.

Figure 7 Blood pressure, cortical blood flow, ECG, heart rate and $E E G$ recorded during severe anoxia. The lower set of traces continues directly from the upper set. The diamond symbols indicate the occurrence of electromagnetic stimulation and the triangles the occurrence of ulnar nerve stimulation. involving the cortex and somato-sensory nuclei in the brain stem or spinal cord. ${ }^{21}$

The activity evoked in the median nerve by electromagnetic stimulation had characteristics of response (latency and duration) consistent with those obtained with electrical stimulation of sensorimotor cortex ${ }^{22}$ or of the pyramidal tract. ${ }^{23} 24$ In our experiments it cannot be determined if electromagnetic stimulation evoked activity directly in the corticospinal tract, or indirectly, by exciting the sensorimotor cortex. It is, however, remarkable that a single electromagnetic stimulus excites action potentials in motor nerves or muscles, whereas for electrical stimulation of the cortex and pyramidal tract in the cat a train of pulses is needed..$^{22-24}$ The observation that the latency and duration of the responses did not change in the course of each experiment before anoxia implies that repeated, maximum intensity electromagnetic stimulation of the brain did not alter the responsiveness of the motor cortex or the corticospinal tract.

The SEPs recorded in the sensorimotor cortex on stimulation of the ulnar nerve displayed latencies comparable to those previously reported. ${ }^{14}{ }^{25}$ Repeated series of maximum intensity electromagnetic stimulation did not influence the early or late components of the evoked responses, leading to the conclusion that electromagnetic stimulation does not affect
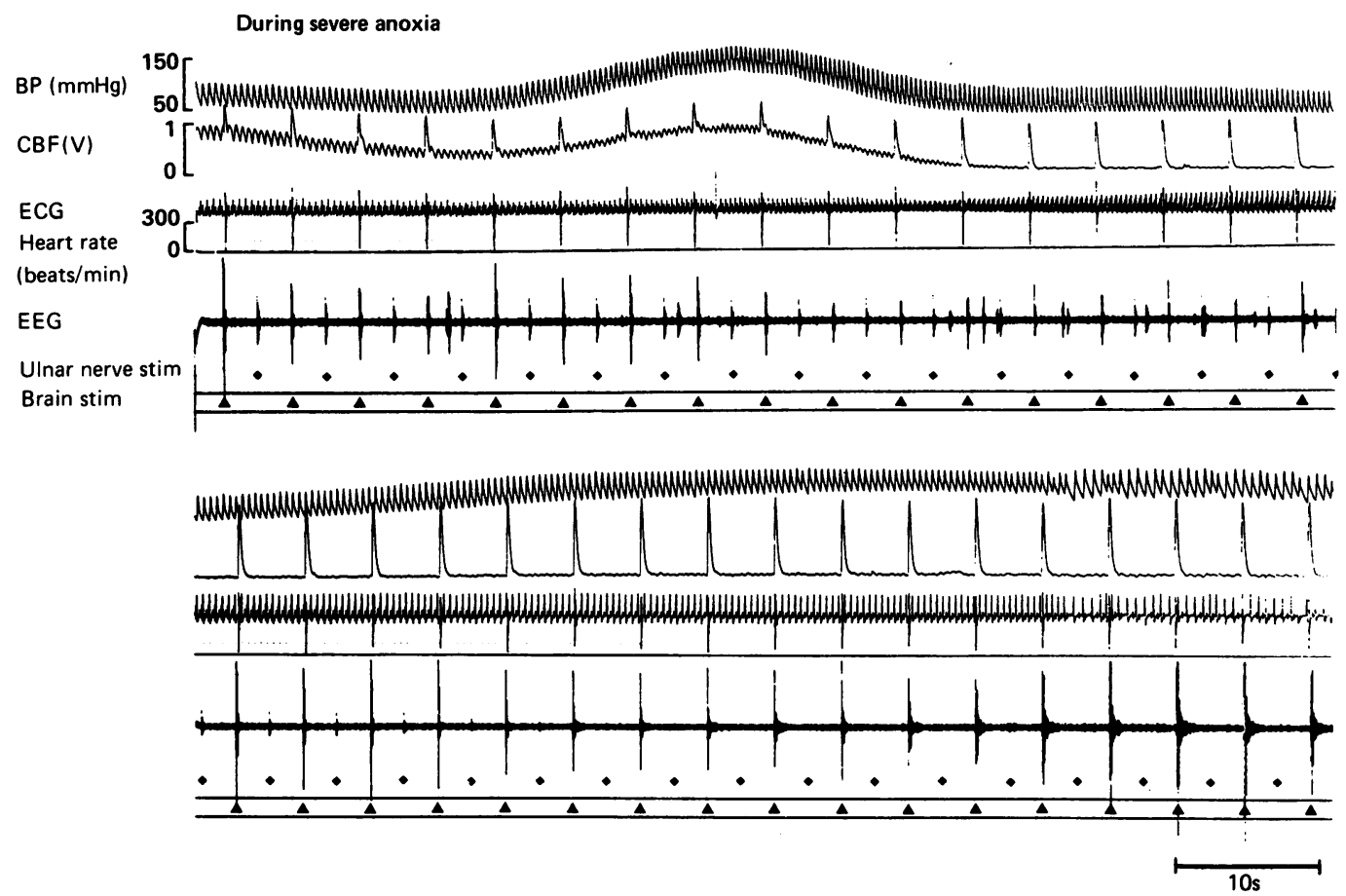

the responsiveness of the sensory cortex to afferent volleys. Following periods of anoxia the SEPs decreased in amplitude, as might be expected, but recovered in spite of continued electromagnetic stimulation.

The calculated maximum charge density induced in the brain with each stimulus (see Methods) was comparable to that reported by Barker et $a^{17}$ in their theoretical discussion of the safety of electromagnetic stimulation of the human brain. These authors argue that the calculated maximum charge density is 100 times less than the threshold for causing neural damage with direct electrical stimulation of the brain as reported by Agnew and McCreery. ${ }^{26}$

In the EEG recordings before and after electromagnetic stimulation, a marked degree of spindle activity was observed. Spindles were commonly observed in rats anaesthetised with propofol, and their occurrence may well be a characteristic of this anaesthetic agent. ${ }^{27}$ There was no change in the background EEG activity with repeated, maximum intensity electromagnetic stimulation of the brain. No evidence of kindling was observed even during or following anoxia, although it should be noted from studies in the rat and the cat that electrical stimuli at $3 \mathrm{~Hz}$, irrespective of the number of stimuli applied, were not able to cause kindling. ${ }^{28}$ (It was not possible to investigate the phenomenon of kindling with electromagnetic 
stimulation because the stimulator used could not deliver stimuli faster than 1 per $5 \mathrm{~s}$. A major concern in the use of electromagnetic stimulation in humans has been the possibility of inducing seizure activity. To date no electromagnetic stimulator is able to deliver stimuli at a rate even approximating that required to induce kindling. Nevertheless, the lack of any epileptogenic activity, even after periods of anoxia, would strengthen the view that the technique, employing the low rates of stimulation of our experiments, is unlikely to induce seizures in humans. However, the studies were of necessity conducted under general anaesthesia and propofol was chosen since it does not have excitatory effects during induction or maintenance of anaesthesia ${ }^{27}$ and has no reported anticonvulsant action. The present results in the cat further support the previous observations by Cohen and Hallett, ${ }^{29}$ who recorded the EEG in adult human subjects during and after prolonged percutaneous electrical stimulation of the brain and who did not show epileptogenic activity.

Cerebral blood flow was measured continuously by a laser Doppler flow method, well established for blood flow measurements in skin, ${ }^{30}$ but only recently evaluated in the brain. ${ }^{15}$ The method is sensitive to the density of the capillaries within the tissue studied and measurements of absolute flow cannot be obtained. In the rabbit, however, a linear relationship has been obtained between cortical blood flow measured with the laser Doppler method and cerebral blood flow measured by radionuclide microspheres over flows of 10 to $200 \mathrm{ml} / \mathrm{min} / 100 \mathrm{~g} .{ }^{15}$ In our experiments no long term changes have been observed in blood flow in the sensorimotor cortex following maximum intensity electromagnetic stimulation either before or after anoxia. In human experiments Ingvar has shown changes in blood flow in regions of the cerebral cortex in association with different voluntary intentions and activities. ${ }^{31-32}$ The lack of changes in cortical blood flow associated with electromagnetic stimulation in our experiments are not necessarily at variance with these observations. In the human experiments the changes in cerebral blood flow occurred in relation to the preparation and execution of complex voluntary activities over several seconds or minutes. In contrast, electromagnetic stimulation is relatively infrequent, its duration is very brief and the motor responses involve only brief involuntary twitches in a few muscle groups.

Measurements of blood pressure and heart rate have been taken to indicate changes in autonomic control exerted by the brain following electromagnetic stimulation. No systematic changes have been recorded, suggesting that large magnetic fields generated in the brain did not significantly perturb autonomic function.

In conclusion, our study in the cat has failed to reveal any acute adverse consequences of electromagnetic stimulation of the brain either under normal respiratory conditions, or during or following repeated anoxia.

We are grateful to the Medical Research Council, and the Chest, Heart and Stroke Association for their support. JAE is a Wellcome Senior Fellow in Clinical Science.

1 Barker AT, Jalinous R, Freeston IL. Non-invasive magnetic stimulation of human motor cortex. Lancet 1985;i1:106-7. 2 Mills KR, Murray NMF, Hess CW. Magnetic and electrical transcranial brain stimulation: physiological mechanisms and clinical applications. Neurosurgery 1987;20:164-8.

3 Hess CA, Mills KR, Murray NMF. Responses in hand muscles from magnetic stimulation of the human brain. $J$ Physiol 1987;388:397-419.

4 Koh THHG, Eyre JA. Maturation of corticospinal tracts by electromagnetic stimulation of the motor cortex. Arch Dis Childhood 1988;63:1347-52.

5 Ingram DA, Thompson AJ, Swash M. Central motor conduction in multiple sclerosis: evaluation of abnorconduction in multiple sclerosis. evaluation of abnormalities revealed by transcutaneous magnetic stimulation of the brai $487-94$.

6 Claus D, Harding AE, Hess CW, Mills KR, Murray NMF, Thomas PK. Central motor conduction in degenerative ataxic disorders: a magnetic stimulation study. $J$ Neurol Neurosurg Psychiatry 1988;51:790-5.

7 Hess CW, Mills KR, Murray NMF, Schriefer TN. Magnetic brain stimulation: central motor conduction studies in multiple sclerosis. Ann Neurol 1987;22:744-53.

8 Caramia D, Bernardi G, Zarola F, Rossini PM. Neurophysiological evaluation of the central nervous propagation in patients with sensorimotor disturbances. Electroencephalogr Clin Neurophysiol 1988;70:16-25.

9 Macdonell RAL, Donnan GA, Bladin PF. A comparison of somatosensory evoked and motor evoked potentials in stroke. Ann Neurol 1989;25:68-73.

10 Barker AT, Freeston IL, Jalinous R, Jarratt JA. Magnetic stimulation of the human brain and peripheral nervous system: an introduction and the results of an initial clinical evaluation. Neurosurgery 1987;20:100-9.

11 Kandler RH, Jarratt JA, Gumpert EJW, Davies-Jones GAB, Venables GS, Sagar HJ. Magnetic stimulation as a quantifier of motor disability. J Neurol Neurosurgery Psychiatry 1989;52:1205.

12 Eyre JA, Flecknell PA, Kenyon BR, Koh THHG, Miller S. The effects of electromagnetic stimulation of the brain on cortical activity, cortical blood flow, blood pressure and heart rate in the cat. J Physiol 1988;396:154P.

13 Miller S, Reitsma DJ, van der Meché FGA. Functional organisation of long propriospinal pathways linking
lumbo-sacral and cervical segments in the cat. Brain Research 1973;62:169-88.

14 Wolsey CN. Organization of somatic sensory and motor areas of the cerebral cortex. In: Harlow HF, Woolsey CN, eds. Biological and biochemical bases of behaviour. Madison: University of Wisconsin Press. 1958:63-81.

15 Eyre JA, Essex TJH, Flecknell PAy Bartholomew PH, Sinclair JI. A comparison of measurements of cerebral blood flow in the rabbit using laser Doppler spectroscopy and radionuclide labelled microspheres. Clin Phys Physiol Meas 1988;9:65-74.

16 Byrne PO, Eyre JA, Kenyon BR, Koh THHG, Miller S, Oliver PD. High current discharge circuit for electromagnetic stimulation of the brain in man. J Physiol 1987;391:3P.

17 Barker AT, Freeston IL, Jalinous R, Jarratt JA. Magnetic and electrical stimulation of the brain: safety aspects. In: Rossini PM, Marsden CD, eds. Non-invasive stimulation of brain and spinal cord: fundamentals and clinical applications. New York: Alan R Liss, 1988:131-44.

18 Barker AT, Eyre JA, Kenyon BR, Koh THHG, Miller S, Wraith $J$. Blocking of transmission of stimulus-related electrical interference to inputs of high-gain a.c. preamplifiers used for neurophysiological measurement. J Physiol 1987;391:13P.

19 Amassian VE, Quirk GJ, Stewart M. Magnetic coil versus electrical stimulation of monkey motor cortex. J Physiol 1987;394:119P.

20 Edgley SA, Eyre JA, Lemon RN, Miller S. Excitation of the corticospinal tract by electromagnetic and electrical stimulation of the scalp in the Macaque monkey. J Physiol 1990) (In press).

21 Gordon G, Jukes MGM. Descending influences on the exteroceptive organisation of the cat's gracile nucleus. $J$ Physiol 1964;173:291-319.

22 Lundberg A, Voorhoeve P. Effects from the pyramidal tract on spinal reflex arcs. Acta Physiol Scand 1962;56:201-9.

23 Lloyd DPC. The spinal mechanism of the pyramidal system in cats. $J$ Neurophysiol 1941;4:525-46.

24 Porter R, Phillips CG. Corticospinal neurones. Their role in movement. London: Academic Press, 1977:111-7.

25 Marshall WH, Woolsey $\mathrm{CN}$, Bard P. Observations on cortical somatic sensory mechanisms of cat and monkey. $J$ Neurophysiol 1941;4:1-24.

26 Agnew WF, McCreery DB. Considerations for safety in the use of extracranial stimulation for motor evoked potentials. Neurosurgery 1987;20:143-7.

27 Glen JB. Animal studies of the anaesthetic activity of ICI 35 868. Br J Anaesthet 1980;52:731-41.

28 Goddard GV, McIntyre DC, Leech CK. A permanent change in brain function resulting from daily electrical stimulation. Exper Neurol 1969;25:295-330.

29 Cohen LG, Hallett $M$. Non-invasive electrical stimulation of the brain does not cause short-term changes in the electroencephalogram. In: Rossini PM, Marsden CD, eds. Non-invasive stimulation of brain and spinal cord: eds. Non-invasive stimulation of brain and spinal cord: fundamentals and clinic

30 Nilsson GE, Tenlandt T, Oberg PA. Evaluation of laser Doppler flow meter for measurement of tissue blood flow. IEEE Trans Biomed Engineering 1980;BME-27:597-604.

31 Ingvar DH, Philipson L. Distribution of cerebral blood flow in the dominant hemisphere during motor ideation and motor performance. Ann Neurol 1977;2:230-7.

32 Ingvar DH. Functional landscapes in the brain studied with regional blood flow measurements. Br J Radiol 1978; 51:657-64. 